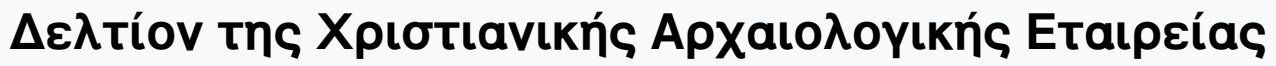

Tóp. 4 (1939)

$\Delta \varepsilon \lambda$ tíov XAE 4 (1936-1938), Пврі́обос Г'

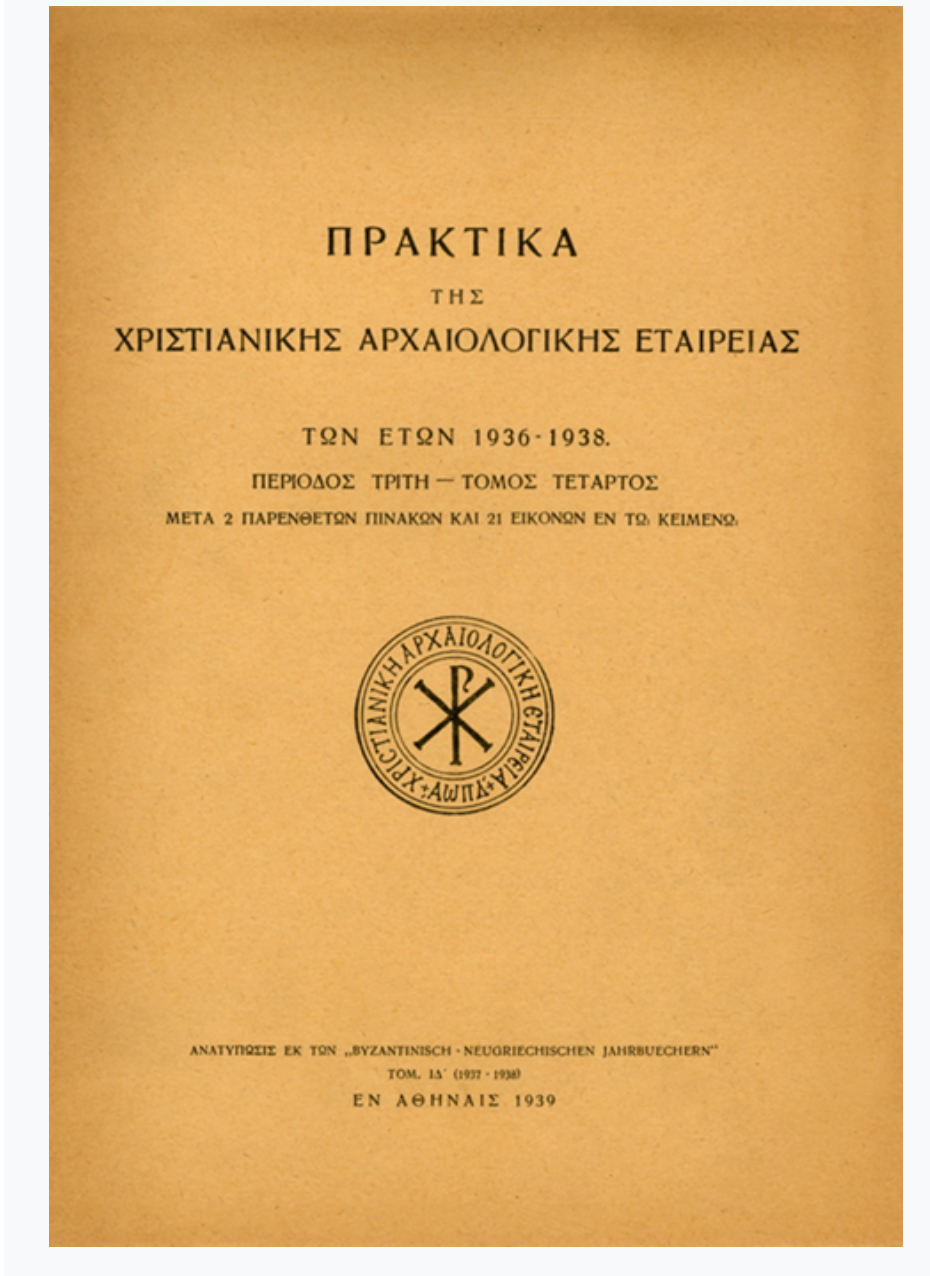

Ökumenios der Apokalypsen-Ausleger und Ökumenios der Bischof von Trikka

Josef Schmid DILLINGEN

doi: $\underline{10.12681 / \text { dchae. } 1423}$

\section{$\triangle \varepsilon \lambda T(0 V X A E 4(1936-1938)$, Пeplodos T'}

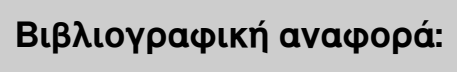

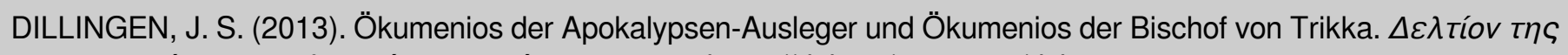

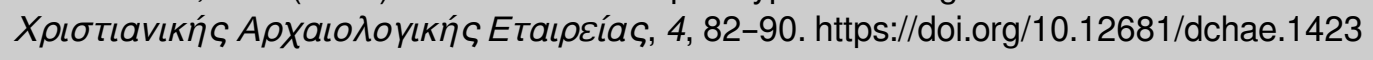




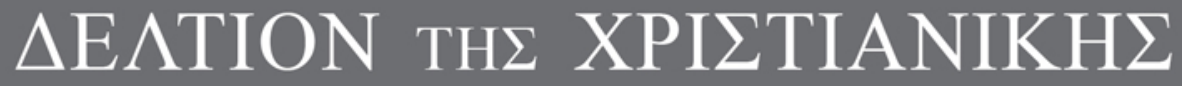 APXAIO $\Lambda$ OГIKH $\Sigma$ ETAIPEIA $\Sigma$}

Ökumenios der Apokalypsen-Ausleger und Ökumenios der Bischof von Trikka

Josef Schmid DILLINGEN

$\Delta \varepsilon \lambda \tau$ tíov XAE 4 (1936-1938), Пв@íodos $\Gamma^{\prime} \bullet \Sigma \varepsilon \lambda .82-90$

A@HNA 1939 


\title{
ÖKUMENIOS DER APOKALYPSEN-AUSLEGER UND ÖKUMENIOS DER BISCHOF VON TRIKKA
}

\author{
JOSEF SCHMID \\ DILLINGEN (DONAU) *
}

In einem ergebnisreichen Aufsatz über die Schriftstellerei der Erzbischofs Antonios von Larissa hat der Herausgeber dieser Zeitschrift (Bd. I2, Jg. 1935/36, S. 300-319, bes. 317-319) auch einen wichtigen Beitrag geliefert zur endgültigen Aufhellung des Dunkels, das über der Persönlichkeit des byzantinischen Exegeten Ökumenios lag. Seine Lebenszeit, sein theologischer Standpunkt und seine schriftstellerische Leistung waren allerdings bereits durch die Forschung der letzten Jahrzehnte, vor allem durch F. Diekamp bestimmt greifbar geworden. Unklar war aber bisher noch, wie er in einem Teil der Überlieferung den Titel eines Bischofs von Trikka in Thessalien (dem heutigen Trikkala) bekommen konnte. Unter den von Bees untersuchten Panegyriken des Antonios von Larissa (Mitte

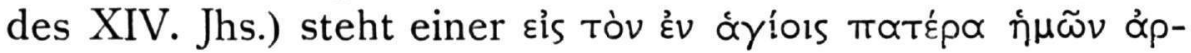

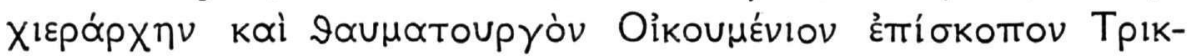
$\kappa \dot{\alpha} \lambda \omega \nu$, worin dieser als eine strahlende Leuchte des Erdkreises bezeichnet wird. Ob der Panegyrikos auch für unsere genauere Kenntnis der Lebensschicksale dieses Mannes ergiebig ist, kann vor der Verröffentlichung des Textes noch nicht gesagt werden. Sein Verfasser scheint von dem

* Denkschrift der Christlich-Archäologischen Gesellschaft zu Athen durch Prof. Dr. N. A. Bees vorgelegt. 
Bischof Ökumenios durch eine Zeit von vier Jahrhunderten getrennt zu sein. Die Gestalt des letzteren wird darum bereits durch die Tradition jener vier Jahrhunderte ihre bestimmt umrissene Prägung erhalten haben. Aus dem von Bees beigebrachten Material aus der Tradition Thessaliens gewinnen wir jedenfalls ein hinreichend klares Bild von der Verehrung, welche dieser Bischof als Heiliger in der griechischen Kirche durch die Jahrhunderte genossen hat. Nach den thessalischen Lokalüberlieferungen hat er um die Mitte des X. Jhs. gelebt und seine Persönlichkeit ist geschichtlich fest umrissen: er ist ein Mann des hohen Mittelalters, der in der Überlieferung und im Kult der griechischen Kirche als Bischof, "heiliger Kirchenvater" und Wundertäter fortlebt und dessen Verehrung nicht auf Thessalien beschränkt geblieben ist.

Neben diesem Bischof Ökumenios steht ein anderer, über den wir zwar auch nur Weniges wissen, aber doch genügend, um uns ein bestimmtes Bild von ihm machen zu können. Stellen wir das Sichere kurz zusammen: Er ist $\mathrm{nicht}$ der Verfasser jenes grossen Scholienwerkes zur Apg, den Katholischen und Paulusbriefen, als der er jahrhundertelang gegolten hat. Wohl aber bleibt sein Name fest verbunden mit dem ältesten auf uns gekommenen griechischen Apokalypsen-Kommentar, den F. Diekamp entdeckt und H. C. Hoskier herausgegeben hat. Es ist ferner auch kaum mehr ein $Z$ weifel erlaubt, dass er nicht bloss, wie sein Kommentar beweist, in seinem theologischen Standpunkt ein Anhänger des Monophysiten Severos von Antiochien ( $† 538)$, sondern auch sein unmittelbarer Zeitgenosse und Korrespondent war, womit auch seine Lebenszeit bestimmt ist. Die Überlieferung seines Kommentars nennt ihn "Philosoph und Rhetor", eine andere Quelle "Scholastikos" und „Komes von Isaurien“, weiss aber nichts davon, dass er Bischof war. Andreas von Kaisareia, der bald nach ihm wieder die Apk ausgelegt hat, setzt sich, wenn er auch seinen 
Namen nie nennt, beständig mit seinem Kommentar auseinander und bestätigt damit, dass Ökumenios in der ersten Hälfte des VI. Jhs. gelebt hat. Soweit scheint kein Problem mehr vorhanden zu sein. Die zwei Träger des einen Namens Ökumenios stehen bestimmt und klar von einander unterschieden vor uns. Unerklärt war dagegen bis jetzt, wie der Apokalypsen - Kommentator Ökumenios in einem bestimmten Teil der Überlieferung zum Bischof von Trikka werden konnte. Bees schreibt dazu: ${ }^{1}$ "Wie sich nun der Laie Ökumenios, der Apokalypsen - Kommentator, zu einem Bischof von Trikka in Thessalien gewandelt hat, bleibt ein Rätsel. Man könnte annehmen, Ökumenios, der Apokalypsen-Kommentator, sei mit dem gleichnamigen Bischof von Trikka in Thessalien verwechselt worden“. Und er fügt hinzu, ein Bischof dieser thessalischen Stadt namens Ökumenios lasse sich vor dem X.-XI. Jh. auf Grund von Urkun. den oder anderen zuverlässigen Quellen nicht nachweisen.

Dazu möchte ich meinerseits einige Bemerkungen fügen und zu zeigen versuchen, dass die von Bees ausgesprochene Vermutung tatsächlich die grösste innere Wahrscheinlichkeit besitzt und eine andere Lösung des genannten Rätsels daneben nicht in Betracht kommt. Ich führe zunächst das handschriftliche Material an, auf das sich die Bezeichnung des im VI. Jh. lebenden Apokalypsen - Auslegers Ökumenios als Bischof von Trikka in Thessalien stützt. Es handelt sich dabei um sechs Hss, die sich aber sofort auf bloss vier verringern werden.

In einer Gruppe des sogenannten $\mathrm{K}$ - Textes der Apk, die aus fünf Hss besteht (sie tragen in Gregorys Liste der griechischen Hss des NT die Nummern: 203-452-467-506202I, bei Hoskier ${ }^{2}$, die Nummern: I07-42-53-26-4I) ist als Ersatz für die bei der Apk sonst fehlende úmó̧દoıs der Prolog zum Apk - Kommentar des Arethas vorangestellt. In

1) A. a. O., S. 318 b.

2) Concerning the Text of the Apocalypse. 2 Bde. London 1929. 
dreien von diesen, die auch in der Textform eine engere Gruppe bilden, nämlich in $452 \cdot 46_{7}-2021$, ist diesem Arethasprolog nochmals ein Text vorangestellt, der die Überschrift trägt:

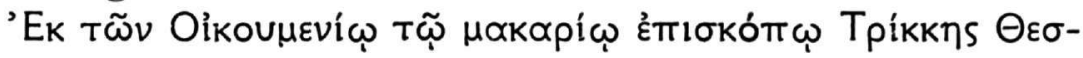

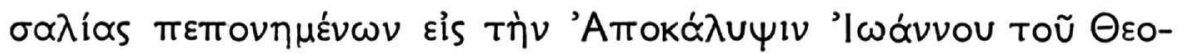

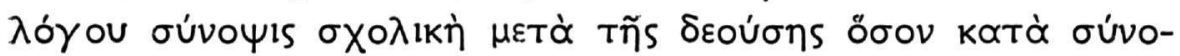

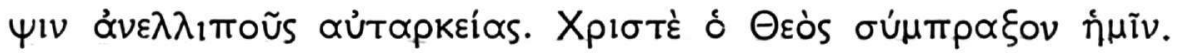

Von den genannten drei Hss sind zwei, nämlich 467 (Paris, B. N. gr. 59) und 202 I (Vatic. Regin. gr. 68) von dem bekannten Georgios Hermonymos, der seit $\mathrm{I}_{472}$ in Paris Griechisch lehrte, aus e i n e r Vorlage abgeschrieben worden, und zwar aus der dritten der genannten Gruppe (452 $=$ Vatic. Pii II gr. 50). Es ist dies eine Pergamenths des XII. Jhs. mit ${ }_{327}$ Blättern, die den Praxapostolos und die Apk ohne Kommentar enthält. Den Nachweis, dass 467 und 202 I aus $45^{2}$ abgeschrieben wurden, glaube ich in Biblica ${ }_{7} 7$ (1936) I79- I82 erbracht zu haben.

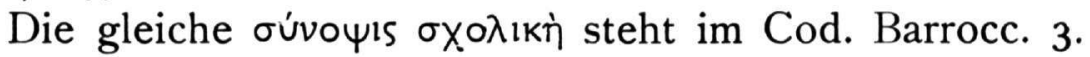
der Bodleiana in Oxford (Gregory 314, Hoskier 6), und zwar ist sie hier dem Kommentar des Arethas vorangestellt. Infolge Verlusts eines Blattes fehlt die erste Hälfte der "Synopse" und damit auch die Überschrift mit dem Namen des Bischofs Ökumenios von Trikka'. Es fehlt aber jeder Grund zu einem Zweifel daran, dass die Überschrift hier ebenso gelautet hat wie in den vorgenannten Hss. Denn alle übrigen Hss, die diesen Text überhaupt bieten, bringen ihn auch unter dem Namen des Bischofs Ökumenios.

An dritter Stelle ist zu nennen Athen, Nationalbibl. 490 (Gregory 254, Hoskier 25I), eine Papierhs des XV. Jhs. mit 906 Seiten, die den Praxapostolos und die Apk mit Kommentar enthält, und zwar zur Apk dem des Andreas.

1) Vgl. J. A. Cramer, Catenae Graecorum Patrum in NT VIII. Oxford 1844. S. $173 \mathrm{f}$. 
Der Schreiber der Hs hat aber in einer etwas naiven Weise bewiesen, dass er aus dem Vollen schöpfen konnte und einen reichen Bestand an Hss zur Verfügung hatte. Er hat nämlich dem Andreas - Kommentar eine ganze Reihe von Stücken anderer Herkunft vorangestellt. An die Spitze stellt er unsere "Synopse“, von der er allerdings nur das erste Drittel abgeschrieben hat. Dann folgt der Pinax und die Überschrift zum Kommentar des Arethas, dahinter ein Text, der in einigen anderen Andreashss am Schluss des Kommentars steht, und dann endlich der Kommentar des Andreas selbst. Sollte es blosser Zufall sein, dass in allen bisher genannten Hss auf die "Synopse“ entweder der ganze Kommentar des Arethas oder dessen Prolog oder der Pinax und die Überschrift dazu folgt, obgleich die "Synopse" natürlich nicht zum Arethas-Kommentar gehört ?

Nun komme ich endlich zu jener $\mathrm{Hs}$, die insofern die wichtigste von allen ist, als durch sie die „Synopse“ zuerst bekannt ${ }^{1}$ und verschiedene Irrtümer über Ökumenios und seinen Apokalypsen-Kommentar veranlasst wurden. Es ist dies der Cod. Coisl. gr. 224 der Pariser Nationalbibliothek, eine schöne Pergamenths des XI. Jhs. mit 378 Blättern, welche den Praxapostolos und (fol. 334-373) die Apk mit Kommentar enthält. Der Kommentar zur Apk, den Cramer darnach veröffentlicht hat, weil er ihn für den des Ökumenios hielt, hat mit Ökumenios nichts zu tun, sondern ist eine verkürzte Fassung des Andreas - Kommentars. Er ist auch in einigen anderen Hss erhalten ${ }^{2}$. In diesem Cod. Coisl. 224 steht nun fol. 330 auch die Synopse. Es war ein Missverständnis Cramers, dass er aus der Stellung der Synopsis vor diesem verkürzten Andreas-Kommentar (der keine eigene Überschrift trägt) geschlossen hat, die Synopse gehöre wirk-

1) B. de Montfaucon hat sie zuerst abgedruckt in seiner Bibliotheca Coisliniana. Paris 1715. S.277-79. Daraus hat sie Migne, PG 119, Sp. 721-26 übernommen.

2) Vgl. J. Schmid, Der Apokalypsetext des Arethas von Kaisareia. (Texte und. Forschungen zur byzantinisch-neugriechischen Philologie. Nr. 17.) Athen 1936. S. 27 ff., wo der Apk-Text dieser Gruppe behandelt ist. 
lich als Prolog zu diesem ${ }^{1}$. Dass dies nicht die Meinung des Schreibers der Hs selbst war, dass er sie vielmehr bloss deshalb dorthin stellte, weil ihm dies ein halbwegs passender Platz für dieses Stück zu sein schien, kann man daraus ersehen, dass er der Synopse noch einen anderen Text unbekannter Herkunft vorangestellt hat, in dem gegen anders Denkende dargelegt wird, dass die Apk wirklich vom Apostel und Evangelisten Johannes stammt ${ }^{2}$.

Irgendeine andere $\mathrm{Hs}$, welche dieses Stück enthielte oder ein anderer Text, der dem Apokalypsen-Ausleger Ökumenios den Titel eines Bischofs von Trikka gäbe, ist mir bei meinen Untersuchungen der griechischen Apk - Hss nicht begegnet. Damit ist gesagt, das s e in zig und a llein der Verfasser dieser Synopsis den Exegeten und den Bischof identifiziert. Man kann deshalb nicht etwa von einem bestimmten Traditionsstrom sprechen, in dem diese Gleichsetzung erfolgt, sondern nur von dem "Zeugnis" oder richtiger der Meinung e in es e inzelne n.

Um den Wert oder Unwert dieses ganz isolierten Zeugnisses vollends würdigen zu können, ist nach seinem A l t e r zu fragen. Dabei ist von der Beobachtung auszugehen, dass die Überschrift der Synopsis unverkennbar der Überschrift nachgebildet ist, die Arethas seinem Kommentar gegeben hat. Ich setze die letztere, um einen bequemen Vergleich der beiden Texte zu ermöglichen, im vollen Wortlaut hierher.

1) Cramer (a.a.O.iv.vi) hat nicht bloss die „Synopse“ als das Proömium zu dem folgenden Kommentar angesehen (dessen Verhältnis zu Andreas er nicht gesehen hat) und diesen deshalb dem Oekumenios zugeschrieben. Er hat aus der Beobachtung, dass das gleiche „Proömium" auch im Cod. Bodl. Barocc. 3 vor dem Arethas-Kommentar steht, den weiteren irrigen Schluss gezogen, die Synopsis gehöre wlrklich zu beiden Kommentaren und der (Arethas-) Kommentar der Oxforder Hs sei eine Zusammenarbeitung der Kommentare des Andreas, Arethas und Oekumenios („Scholia Barocciana... conflata sunt, ut videtur, ex Andreae, Arethae et Oecnmenii commentariis").

2) Abgedruckt bei Montfaucon, a. a. O., S. $276 \mathrm{f}$. 


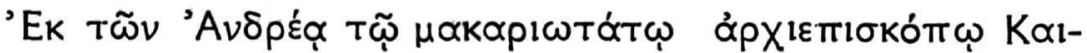

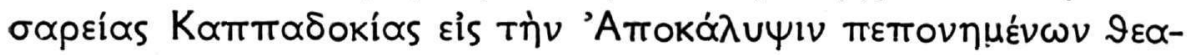

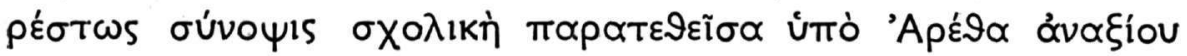

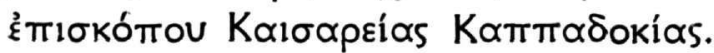

Mit dieser Beobachtung ist ein fester terminus post quem für die Abfassung der Synopsis gewonnen. Weil Arethas seinen Kommentar um 895 geschrieben hat, ist sie frühestens um 900 entstanden. Sie ist aber auch nicht sehr viel jünger, weil sowohl der Cod. Coisl. 224 als auch der Barocc. 3, die sie enthalten, dem XI. Jh. angehören. Der Zeitraum für ihre Entstehung ist damit auf ungefähr ein Jahrhundert oder ein wenig mehr eingeschränkt.

Inhaltlich ist die Synopsis, wie auch die Überschrift sagt, eine kurze Zusammenfassung einiger Hauptgedanken, die Ökumenios auf den ersten Seiten seines Kommentars ausspricht ${ }^{1}$. Daraus ergibt sich mit voller Klarheit, dass ihr Verfasser, wenn er den Namen des Ökumenios nennt, wirklich den Apokalypsen-Ausleger des VI. Jh. meint. Ob sein "Werk" ein Fragment ist in dem Sinne, dass er vielleicht im Sinne hatte, den vollständigen Kommentar des Ökumenios in der genannten Weise zu bearbeiten, kann hier auf sich beruhen bleiben. Wahrscheinlich hat er überhaupt nicht mehr leisten wollen, als uns erhalten ist. Die entscheidend wichtige Frage, die wir nun zu stellen haben, ist die, ob er etwa auf Grund guter geschichtlicher Kenntnisse bezeugt, dass der Severianer des VI. Jhs. auch Bischof von Trikka in Thessalien geworden ist, oder ob ihm nicht vielmehr der eine, einer ziemlich weit zurückliegenden Vergangenheit angehörige Träger des Namens Ökumenios mit dem ihm

1) Vgl. H. C. Hoskier, The complete Commentary of Oecumenius on the Apocalypse. Ann Arbor 1928. S. 29-37. Fast wörtlich gleich ist neben der Aufzählung der alten Autoritäten für die Echtheit und Kanonizität der Apk und dem wörtlichen Zitat von Kol 1, $16 \mathrm{der}$ Satz (Cramer VIII 175, $1 \mathrm{ff}$. = Hoskier 32): ì

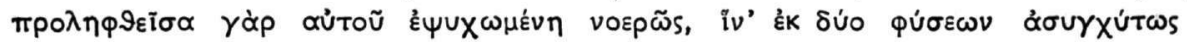

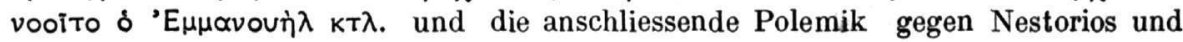
Eutyches. 
zeitlich viel näherstehenden Bischof und Wundertäter zu einer Person zusammengeflossen ist. Wir können die erste dieser beiden Möglichkeiten wohl nicht absolut ausschliessen. Sie verliert aber doch praktisch alle Wahrscheinlichkeit, sobald man das absolute Schweigen der übrigen Überlieferung über die Bischofswürde des Severianers Ökumenios danebenhält. Weder die Überschrift zu seinem Kommentar noch das syrische Florileg, das ein Stück des Kommentars verwertet hat, noch endlich der fast gleichzeitige Liberatus von Karthago ${ }^{1}$, der von ihm spricht, weiss etwas von seiner bischöflichen Würde. Auf der anderen Seite bereitet die Annahme nicht die geringste Schwierigkeit, dass dem Verfasser der Synopsis eine Verwechslung unterlaufen ist. Glaubte er wirklich in dem Autor des Kommentars, den er zur Hand hatte, den einer späteren Zeit angehörigen Bischof vor sich zu haben, dann konnte ihn auch nicht die anders lautende Überschrift des Kommentars davon abhalten, ihm den Titel „Bischof von Trikka“ beizulegen. Der „Philosoph und Rhetor" musste dann dem gewichtigeren Titel „Bischof“ weichen.

Die Haltbarkeit dieser in sich wahrscheinlichen Hypothese hängt noch von einer weiteren Voraussetzung ab, dass nämlich der Bischof und Wundertäter Ökumenios zum mindesten ein Zeitgenosse des Autors der Synopsis war. Sie würde noch erheblich an innerer Wahrscheinlichkeit gewinnen, wenn er bereits einige Zeit vor ihm gelebt hätte. Träger eines bekannten und angesehenen Namens muss er in jedem Fall bereits gewesen sein. N. J. Giannopoulos und P. Uspenskij haben die Lebenszeit des Bischofs und Wundertäters Ökumenios in die Mitte oder gegen das Ende des X. Jhs. datiert ${ }^{2}$. Nachdem auch die Synopsis wahrscheinlich

1) Vgl. Acta Conciliorum Oecumenicorum, ed. E. Schwartz, vol. II, tom. V . Berlin 1939. S 138, 18-21.

2) Wenn ihn, wie Bees, a.a.O., 318 b, Anm. 2 bemerkt, einige spätere Ako- 
in dasselbe X. oder in den Anfang des XI. Jhs. zu datieren ist, so mündet das hier behandelte Problem schliesslich in die Frage nach der Lebenszeit des wirklichen Bischofs von Trikka in Thessalien aus.

\section{Eine weitere Wandmalerei des ÖKumenios von Trikka.}

In den B.-Ng. Jb., Bd. XII (1935-36), S. $3^{18^{a}}$ ist von Wandmalereien und anderen Kunstwerken, die sich auf den heiligen Ökumenios von Trikka beziehen, die Rede. Ich ergreife die Gelegenheit, auf eine weitere Wandmalerei dieses Heiligen aufmerksarn zu machen. Sie befindet sich in der Hauptkirche des Kato-Panagia-Klosters (bei Arta) und soll aus der Mitte des XIV. Jhs. stammen.

Herr Kollege A. Orlandos hat eine gelungene Abbildung der interessanten Wandmalerei veröffentlicht ${ }^{1}$.

Athen-Berlin .

Nikos A. Bees (Bén૬)

luthien zum Zeitgenossen Konstantins d. Gr. und Teilnehmer am Konzil von Nikäa machen, so ist das natürlich nicht als historische Kunde zu betrachten.

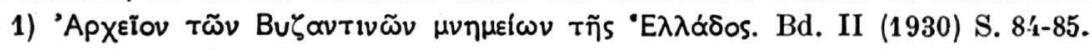

